\title{
POSITRON ANNIHILATION STUDIES OF STYRENE-N-X-PHENYLMALEIMIDES
}

\author{
A. Baranowski, M. Dębowska, K. Jerie, J. Rudzińska-Girulska \\ Institute of Experimental Physics, Wrocław University \\ Maxa Borna 9, 50-204 Wroclaw, Poland
}

\section{Z. PAWElKa}

Institute of Chemistry, Wrocław University, Joliot Curie 14, 50-383 Wrocław, Poland

\section{AND R.T. SIKORSKI}

\begin{abstract}
Institute of Organic and Polymer Technology, Technical University of Wroclaw Wybrzeże Wyspiańskiego 27, 50-370 Wrocław, Poland
\end{abstract}

Results of angular correlation of annihilation radiation and positron annihilation lifetime measurements are presented for five styrene copolymers: poly(co-styrene-phenylmaleimide) and its three derivatives with chlorine as well as for one with the $\mathrm{OH}$ group substituted in the benzene ring. It occurs that the chlorine substituted in three different positions in the benzene ring poly(co-styrene-o (or $-m,-p$ )-chlorophenylmaleimide) inhibits the formation of the positronium to different extent. The greatest effect is observed in case of the chlorine atoms substituted in the benzene ring at ortho-position towards the nitrogen atom. In addition, electric dipole moments were measured for N-X-phenylmaleimide units, to check whether their magnitude influences the positron annihilation parameters. On the basis of the present results no correlation between the positron annihilation parameters and the electric dipole moments of the units was noticed.

PACS numbers: 71.60.+z, 78.70.Bj, 81.20.Sh, 82.30.Hk

\section{Introduction}

Influence of the chlorine (in the gaseous or in the ionic form) on the annihilation characteristics is well known. It was shown $[1,2]$ that in chloropolystyrenes the positronium was inhibited and that the reactivity of chlorine atoms towards the positron depended on its position in the polymer structure. The chlorine in the aliphatic ring inhibited formation of the positronium more effectively than that one in the aromatic ring. Intensities $I_{3}$ of the long-lived component in the positron lifetime spectra vs. the chlorine content $(C)$ for the samples were well 
fitted with the equation $I_{3}=I_{3}^{0}\left[1+(\alpha x C)^{\beta}\right]^{-1}$, where $I_{3}^{0}$ denoted the $o$-Ps yield in the polystyrene. The mentioned equation had been proposed previously [3] to represent the 0 -Ps yield versus the concentration of electron scavengers in solutions.

According to the spur model the Ps yield in polymers should be large due to the small size of the spur in solids at all, and the high values of dielectric constants in polymers. Relative high contributions of the spur mechanism in the Ps formation were estimated in [4] for some polymers.

In case of samples containing $\mathrm{Cl}$ the inhibition of the positronium in the frame of the spur model was frequently explained by the possibility of the $\left[\mathrm{Cl}^{-}, \mathrm{e}^{+}\right]$bound state formation. In [1] the increase in the electron charge in vicinity of chlorine atoms was suggested as a result of the inductive effect following the introduction of chlorine into chloropolystyrenes. It is known that introduction of chlorine enlarges the dipole moment of molecules. Polar molecules with the electric dipole moment greater than $1.625 \mathrm{D}$ are guaranteed to possess positron complexes which are stable with respect to positron detachment [5]. It was suggested [1] that in case of chloropolystyrenes one could take into account the possibility of existence of the positron-unit complex $\left[\mathrm{RCl}, \mathrm{e}^{+}\right]$possible to view as $\left[\mathrm{Cl}^{-}, \mathrm{e}^{+}\right]$'complex in the field of an $\mathrm{R}^{+}$ion. The influence of the electric dipole moment of molecules on positronium formation was reported in [6].

In the present paper results of further studies of the chlorine influence on positron annihilation characteristics are given for samples of styrene- $\mathrm{N}$-phenylmaleimides with $\mathrm{Cl}$ substituted in the benzene ring, together with information on values of the dipole moment of the $\mathrm{N}$-X-phenylmaleimide unit.

\section{Experimental}

Samples of five styrene copolymers: poly(co-styrene-phenylmaleimide) sample No. 1, poly (co-styrene-o-chlorophenylmaleimide) - sample No. 2, poly(co-styrene- $m$-chlorophenylmaleimide) - sample No. 3, poly(co-styrene-p-chlorophenylmaleimide) - sample No. 4, and poly(co-styrene- $p$-hydroxyphenylmaleimide) - sample No. 5, were studied. The samples were obtained by the direct radical copolymerization of styrene and phenylmaleimide monomers containing substituted aromatic rings [7]. The structure of the sample No. 1 is given in Fig. 1. In the samples Nos. 2, 3 and 4 one chlorine atom was substituted in the benzene ring at ortho, meta, and para position towards the nitrogen atom in the N-phenylmaleimide unit, while in the sample No. 5 one hydroxyl group was substituted at the para position in the segment. Molar ratio of the styrene and the N-X-phenylmaleimide units was 1:1.

The powder of each sample was formed into a disc of the diameter equal to $20 \mathrm{~mm}$ and of the thickness equal to $3 \mathrm{~mm}$, under the pressure of 3 tons.

Angular correlation of annihilation radiation (ACAR) and positron annihilation lifetime (PAL) techniques were used in our studies of samples. The two kinds of measurements were performed in air at room temperature.

ACAR measurements were performed from -29 to $+29 \mathrm{mrad}$ with the use of the LS spectrometer of angular resolution with FWHM equal to $0.8 \mathrm{mrad}$, described in details in [8]. Data were accumulated with the step of $0.5 \mathrm{mrad}$ for 


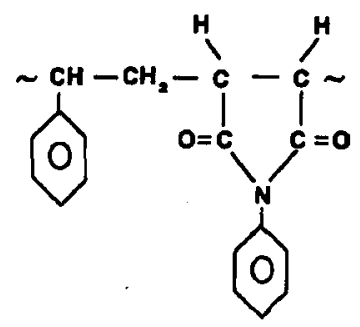

Fig. 1. The structure of the poly(co-styrene-phenyl maleimide).

the period of $3 \times 10^{3} \mathrm{~s}$ at each point resulting in about $6 \times 10^{4}$ counts at the peak at each distribution with the background intensity reaching $3 \%$ of the peak value. The positrons were produced upon desintegration of ${ }^{22} \mathrm{Na}$ nuclei in the source of $15 \mathrm{mCi}$ activity.

PAL measurements were performed with the use of a conventional fast-fast coincidence system with cylindrical $\mathrm{BaF}_{2}$ scintillators $(38 \mathrm{~mm} \times 25 \mathrm{~mm})$ and XP2020Q photomultipliers. The positron source, ${ }^{22} \mathrm{Na}$ of activity $5 \mu \mathrm{Ci}$ was deposited between two identical kapton foils (1 $\mathrm{mg} \times \mathrm{cm}^{-2}$ each) and then sandwiched between the samples under investigation. The geometry of the measurements was "the summing-safe" one, i.e. the sample-source sandwich was placed out of the volume between the scintillators. A typical decay curve contained about $10^{7}$ counts and had the peak to background ratio of 1400 .

The electric dipole moments were estimated for $\mathrm{N}$-X-phenylmaleimide units. Further details of measurements are given in Appendix.

\section{Results}

The ACAR curves were fitted with a sum of three Gaussians, simultaneously together with a background represented as a sloped straight line using the computer program FER [9]. The results of the analysis, i.e. the FWHM and the intensity of each component are given in Table $\mathrm{I}$.

TABLE I

Results of ACAR measurements.

\begin{tabular}{c|c|c|c|c|c|c}
\hline \hline \multirow{2}{*}{$\begin{array}{c}\text { Sample } \\
\text { no. }\end{array}$} & \multicolumn{3}{|c|}{ FWIM [mrad] } & \multicolumn{3}{c}{ Intensities [\%] } \\
\cline { 2 - 7 } & $G_{1}$ & $G_{2}$ & $G_{3}$ & $I_{1}$ & $I_{2}$ & $I_{3}$ \\
\hline 1 & $2.83(0.14)$ & $8.91(0.11)$ & $15.7(1.5)$ & $6.5(0.2)$ & $85.2(0.4)$ & $8.3(0.4)$ \\
2 & $2.39(0.24)$ & $8.92(0.05)$ & $21.3(2.3)$ & $2.4(0.1)$ & $93.3(0.3)$ & $4.3(0.2)$ \\
3 & $2.96(0.21)$ & $8.90(0.07)$ & $18.8(2.2)$ & $4.6(0.2)$ & $90.8(0.4)$ & $4.6(0.3)$ \\
4 & $2.47(0.19)$ & $8.87(0.06)$ & $19.5(2.0)$ & $3.4(0.1)$ & $91.8(0.3)$ & $4.8(0.3)$ \\
5 & $3.05(0.12)$ & $9.22(0.07)$ & $17.9(1.3)$ & $6.2(0.1)$ & $87.2(0.3)$ & $6.6(0.2)$
\end{tabular}


The PAL spectra, corrected for positron annihilation in the source (8\%), were fitted with a sum of three exponentials convoluted with the resolution function of the lifetime spectrometer (a sum of two Gaussians) plus a constant background, using the program POSITRON-FIT [10]. The results of the analysis, i.e. the lifetimes and the intensities are given in Table II.

TABLE II

Results of PAL measurements.

\begin{tabular}{c|c|c|c|c|c|c|c|c|c}
\hline \hline Sample & \multicolumn{4}{|c|}{ Lifetimes [ns] } & \multicolumn{4}{|c|}{$\tau$} & \multicolumn{4}{|c}{ Intensities [\%] } \\
\cline { 2 - 5 } \cline { 6 - 8 } no. & $\tau_{1}$ & $\tau_{2}$ & $\tau_{3}$ & $\tau_{4}$ & {$[\mathrm{~ns}]$} & $I_{1}$ & $I_{2}$ & $I_{3}$ & $I_{4}$ \\
\hline 1 & 0.17 & 0.41 & 1.98 & 3.21 & 2.12 & 17.9 & 48.1 & 30.1 & 3.9 \\
& $(0.01)$ & $(0.01)$ & $(0.04)$ & $(0.26)$ & $(0.12)$ & $(0.7)$ & $(0.6)$ & $(1.4)$ & $(1.5)$ \\
2 & 0.18 & 0.41 & 1.93 & 3.6 & 2.06 & 12.2 & 66.7 & 19.5 & 1.7 \\
& $(0.01)$ & $(0.01)$ & $(0.03)$ & $(0.4)$ & $(0.11)$ & $(0.8)$ & $(0.7)$ & $(0.5)$ & $(0.6)$ \\
3 & 0.18 & 0.43 & 1.93 & 3.24 & 2.05 & 18.3 & 52.4 & 26.6 & 2.7 \\
& $(0.01)$ & $(0.01)$ & $(0.04)$ & $(0.33)$ & $(0.12)$ & $(0.8)$ & $(0.7)$ & $(1.1)$ & $(1.3)$ \\
4 & 0.20 & 0.43 & 1.95 & 4.4 & 2.04 & 17.0 & 58.4 & 23.8 & 0.9 \\
& $(0.01)$ & $(0.01)$ & $(0.02)$ & $(0.7)$ & $(0.09)$ & $(1.1)$ & $(1.0)$ & $(0.3)$ & $(0.4)$ \\
$5^{*}$ & 0.20 & 0.42 & 1.97 & 3.8 & 2.10 & 16.0 & 58.3 & 24.1 & 1.7 \\
& $(0.01)$ & $(0.01)$ & $(0.07)$ & $(0.9)$ & $(0.23)$ & $(2.7)$ & $(2.4)$ & $(1.2)$ & $(1.5)$ \\
\hline
\end{tabular}

*Only $1.4 \times 10^{6}$ counts were collected under the spectrum.

The values of electric dipole moments are given in Table III.

TABLE III

Electric dipole moments of

$N$-X-phenylmaleimides.

\begin{tabular}{c|c}
\hline \hline Derivative & $\mu[\mathrm{D}]$ \\
\hline $\mathrm{H}$ & $1.46(0.01)$ \\
$o-\mathrm{Cl}$ & $1.53(0.02)$ \\
$m-\mathrm{Cl}$ & $2.68(0.01)$ \\
$p-\mathrm{Cl}$ & $2.98(0.03)$ \\
$p-\mathrm{OH}$ & $1.68(0.03)$
\end{tabular}

\section{Discussion and conclusions}

It is evident on the basis of the two kinds of measurements performed by us that the positron annihilation mechanism in the solid state poly(co-styrene-phenylmaleimide) and its three derivatives is strongly affected by both the chlorine and 
the hydroxyl group substituted in the benzene ring of the $\mathrm{N}$-X-phenylmaleimide unit. Following the common way of analysis of the ACAR and PAL results obtained for polymers by others and generally accepted, we assume that the intensity of the narrowest Gaussian $\left(I_{1}\right)$ reflects the yield of the para-positronium formed in samples under study. The sum of the intensities $I_{3}+I_{4}$ of the longest-lived components in the PAL spectra is ascribed to the ortho-positronium atoms that were annihilated by pick-off in free volume of samples.

The two components reflect bimodal distributions of the free volume in the samples which will be given elsewhere [11]. However, the population of the larger volume sites by the o-Ps atoms $\left(I_{4}\right)$ is considerably smaller than in case of the second ones connected with $I_{3}$. The average lifetime $\bar{\tau}$ of the two components $\left(\tau_{3}, \tau_{4}\right)$ is given in Table II. Regarding its values as well as the values of $I_{1}$ in Table I and the sum of $I_{3}+I_{4}$ in Table II, one can conclude that the positronium is inhibited in samples with substituents. The greatest effect is observed in case of the chlorine atom substituted at the ortho-position towards the nitrogen atom. We ascribe the latter to the steric reasons (cf. results and discussion in Appendix), while the mechanism of the inhibition in all samples still remains unknown. The inductive effect produced by the chlorine that had been introduced to the polymer structure was assumed by us to be the reason of the inhibition of the positronium formation in chloropolystyrenes [1]. At present no correlation between the electric dipole moment of the $\mathrm{N}$-X-phenylmaleimide unit and any of the annihilation parameters is seen.

\section{Appendix}

The dipole moment (in D) is given by the formula $\mu=0.21986\left(P_{\mathrm{dip}}\right)^{1 / 2}$,

where $P_{\text {dip }}$ denotes the molar dipole polarization of the solute.

$P_{\text {dip }}$ has been calculated according to the expression given by Hedestrand [12]:

$P_{\text {dip }}=\frac{3 M_{1}}{d_{1}} \frac{\alpha \varepsilon_{1}}{\left(\varepsilon_{1}+2\right)^{2}}+\frac{M_{2}-M_{1} \beta}{d_{1}}\left(\frac{\varepsilon-1}{\varepsilon+2}\right)-P_{\text {def }}$.

$P_{\text {def }}$ denotes the deformation polarization and was assumed as equal to the molar refraction, $R_{d}$ :

$$
R_{d}=\frac{3 M_{1}}{d_{1}} \frac{\gamma n_{1}^{2}}{\left(n_{1}^{2}+2\right)^{2}}+\frac{M_{2}-M_{1} \beta}{d_{1}}\left(\frac{n_{1}^{2}-1}{n_{1}^{2}+2}\right) .
$$

The coefficients $\alpha, \beta$ and $\gamma$ are defined as follows:

$$
\begin{aligned}
& \varepsilon_{12}=\varepsilon_{1}\left(1+\alpha x_{2}\right), \\
& n_{12}^{2}=n_{1}^{2}\left(1+\gamma x_{2}\right), \\
& d_{12}=d_{1}\left(1+\beta x_{2}\right) .
\end{aligned}
$$

$x, \varepsilon, n$ and $d$ are mole fraction, dielectric constant, refractive index and density, respectively.

The indexes 1,2 and 12 refer to solvent, solute and solution, respectively. Measurements were performed for solutions of $\mathrm{N}-\mathrm{X}$-phenylmaleimides in dioxane for $x_{2}$ changing from $1 \times 10^{-3}$ to $10 \times 10^{-3}$. 


\subsection{Experiment}

The dielectric constant was measured by the method of the superheterodyne beat at the frequency of $2 \mathrm{MHz}$ and with the use of the DIPOLMETER DMO1. The dielectric capacity was measured with accuracy of $\Delta c / c \leq 1 \times 10^{-4}$.

The density was evaluated pycnometrically with accuracy of $1 \times 10^{-4} \mathrm{~g} / \mathrm{cm}^{3}$. The refractive index for the $D$-line of sodium was measured with an Abbe refractometer with accuracy of $5 \times 10^{-5}$. All measurements were performed at $298 \mathrm{~K}$.

The dioxane was purified by the standard method [13] and stored over sodium and the molecular sieve of type A4.

Derivatives of N-phenylmaleimide were received from the Institute of Organic and Polymer Technology and used directly.

\subsection{Results and discussion}

The dipole moments of $\mathrm{N}-\mathrm{X}$-phenylmaleimides $(\mathrm{X}=\mathrm{H}, o-\mathrm{Cl}, m-\mathrm{Cl}, p-\mathrm{Cl}$, $p$-OH) are given in Table III.

The dipole moments estimated by us for the derivatives with $\mathrm{X}=\mathrm{H}, o-\mathrm{Cl}$ are in good agreement with the ones that had been estimated previously in $\mathrm{CCl}_{4}$ $(\mathrm{H})$ and in dioxane $(o-\mathrm{Cl})$ as equal to 1.40 and $1.37 \mathrm{D}$ [14].

Results of the dielectric and electrooptic studies of the structure of $\mathrm{N}$-arylmaleimides that had been performed up to the present $[14,15]$ showed the conjugation between the indivisible electron pair of the nitrogen and the benzene ring is inconsiderable. The presence of the strong electronegative carbonyl groups in the imide system is the reason of it. On the other side the steric interaction between the ortho substituents and oxygen of the carbonyl groups is enough strong to deflect the benzene ring from the plane of the maleimide cycle.

The angles of the deflection of the plane of the phenyl ring that had been estimated for different derivatives are contained in the range from $15^{\circ}$ to $70^{\circ}[11,15]$.

One can assume that the unit of the poly(co-styrene-X-phenylmaleimide) comprises the imide of the succinic acid substituted with $\mathrm{X}$ and the ethylbenzene. The dipole moments of the $\mathrm{H}, m-\mathrm{Cl}$ and $p$ - $\mathrm{Cl}$ substituted phenylimides of the succinic acid are respectively equal to $1.68,2.94$ and $3.30 \mathrm{D}$ [16].

It is seen that there is a linear correlation between the dipole moments of the imides of the maleic and succinic acids. The last ones are greater of about $0.3 \mathrm{D}$. The dipole moments of the styrene and the ethylbenzene are equal to $0.0 \div 0.3 \mathrm{D}$ and $0.4 \mathrm{D}$ [17], respectively. Thus their contribution to the total dipole moment of the units of the poly(co-styrene-N-X-phenylmaleimides) should not alter the sequence of the dipole moments.

\section{Acknowledgment}

We are grateful to Prof. T. Goworek and Dr. J. Wawryszczuk from Lublin University for measuring the lifetime spectra.

Work supported in part by the MEN, GBW/94/5/IFD.

\section{References}

[1] A. Baranowski, M. Dębowska, K. Jerie, G. Mirkiewicz, J. Rudzińska-Girulska, R.T. Sikorski, J. Phys. IV 3, 225 (1993). 
[2] A. Baranowski, M. Dębowska, K. Jerie, G. Mirkiewicz, J. Rudzińska-Girulska, R.T. Sikorski, Acta Phys. Pol. A 83, 239 (1993).

[3] G. Wikander, O.E. Mogensen, N.J. Pedersen, Chem. Phys. 77, 159 (1983).

[4] A. Bisi, G. Gambarini, L. Zappa, Nuovo Cimento D 2, 1465 (1983).

[5] A.H. Kurtz, K.D. Jordan, J. Chem. Phys. 75, 1876 (1981).

[6] Z. Zhang, Y. Ilo, Y. Tabata, Radiat. Phys. Chem. 36, 285 (1990).

[7] R.T. Sikorski, A. Puszyński, G. Mirkiewicz, Z. Izdebski, Raport SPR nr 11/1989, Instytut Technologii Organicznej i Tworzyw Sztucznych Politechniki Wroclawskiej, Wrocław 1989.

[8] B. Rozenfeld, A. Baranowski, K. Jerie, Nukleonika 19, 817 (1974).

[9] A. Baranowski, Program FER, in a software of the Computer Library of the Section of Applied Nuclear Physics at Wrocław University.

[10] P. Kirkegaard, N.J. Pedersen, M. Eldrup, PATFIT-88: A Data Processing System for Positron Annihilation Spectra on Mainframe and Personal Computers, Ris $\emptyset \mathrm{Na}$ tional Laboratory, 1989.

[11] A. Baranowski, M. Dębowska, K. Jerie, Z. Pawełka, J. Rudziniska-Girulska, to be published in J. Radioanal. Nucl. Chem.

[12] G. Hedestrand, Z. Phys. Chem. B 2, 428 (1929).

[13] L.A. Weissberger, E.S. Proskauer, Techniques of Organic Chemistry, Organic Solvents, Vol. 7, Interscience, New York 1955.

[14] B.A. Arbuzov, L.K. Novikova-Aleksandrova, S.G. Vul'fson, A.N. Vereshchagin, Izv. Akad. Nauk SSSR 5, 1020 (1979).

[15] B.A. Arbuzov, L.K. Novikova-Aleksandrova, S.G. Vul'fson, A.N. Vereshchagin, Izv. Akad. Nauk SSSR 3, 661 (1979).

[16] A. Arcoria, H. Lumbroso, R. Passerini, Bull. Soc. Chim. (France), 754 (1959).

[17] A.L. Mc Clellan, Tables of Experimental Dipole Moments, Rahara Enterprises, El Cerrito 1973, Vol. 2 and 1989, Vol. 3. 